\title{
Redox Active Polymer as a pH Actuator on a Re-Sealable Microfluidic Platform
}

Divya Balakrishnan ${ }^{1,2}$, Mathieu Gerard1', Doriane Del Frari' ${ }^{1}$, Stephanie Girod ${ }^{1}$, Wouter Olthuis² and César Pascual-García1*

${ }^{1}$ Material Research and Technology Department (MRT), Luxembourg Institute of Science and Technology (LIST), 41 Rue du Brill, L-4422 Belvaux, Luxembourg, Europe ${ }^{2}$ MESA+, University of Twente, Drienerlolaan 5, 7522 NB Enschede, The Netherlands

\begin{abstract}
The electrochemical control of molecular concentrations in liquids is quite challenging because of the small ratio of interacting sites between the electrode surface and the number of molecules in the volume. Here we review our recent works aiming the control of proton concentration combining redox self-assembled monolayers with a modified platform. We used Aminothiolphenol, to obtain coatings with quasi-reversible redox states able to exchange protons with the electrolyte at low voltages using different polymerization methods. We studied their charge exchange during different cycles, which would provide us the possibility to use different cycles of reactions controlled by acidity. We achieved the control of proton concentration over the liquid with an efficient design of the microfluidic device to control the diffusion of protons avoiding the reduction of hydrogen at the counter electrode and providing long lasting stability to carry control of chemical reactions during several tenths of minutes. The experiments were carried out in aqueous environment using a $\mathrm{pH}$ fluorescence marker to track the $\mathrm{pH}$ that allowed us to monitor the proton concentration down to $\mathrm{pH} 5$, where the fluorescence molecule lost its sensitivity, while calculations indicate that the $\mathrm{pH}$ can be below 1.
\end{abstract}

Keywords: Microfluidic platform; Electrochemical cell; Miniaturization; $\mathrm{pH}$ control

\section{Introduction}

Redox active self-assembled monolayers (SAMs) are materials capable of exchanging electrons and ions between electrodes and electrolytes that can facilitate the design of devices like sensors, batteries, or chemical switches [1]. In these devices the changes in the liquid make the chemical modifications on the electrodes to provide them some functionality. In other cases, the electrodes can be conceived as actuators to change the chemical environment [2]. The acidity is one of the main factors of the chemical environment facilitating the control of reactions like the synthesis of polymers [3], the activation of enzymes [4] or physical properties like the swelling of polymer hydrogels [5]. The efficiency to control other chemical processes through the electrochemical generated acid will depend on the proton concentration range achievable, the number of reversible cycles that can be repeated and that the applied voltages to generate or uptake protons do not interfere with the other chemical the reactions that want to be controlled. Nevertheless, the control of the proton concentration from electrodes is quite challenging mainly because it is proportional to the surface to volume ratio of the electrodes, which is unfavorable in normal conditions leading to very small changes of acidity.

Different methods to achieve control of the acidity have been explored in literature. The simplest one in water is electrolysis [6], which separates the water molecules into $\mathrm{H}^{+}$and $\mathrm{OH}^{-}$ions at voltages over $1.23 \mathrm{~V}$ (against normal $H^{+}$reference electrode). Though this method is easy to implement, the disadvantage is that it is an irreversible reaction and that the high potentials could trigger some other reactions with the chemicals in solution. To reduce the energy of the reactions and achieve some quasireversibility the electrode surfaces can be coated with redox active conducting polymers like PEDOT or polypyrrole $[7,8]$. The disadvantage is that polymers coatings tend to degrade with repeating reactions through excess of crosslinking showing poor reversibility. The impact of the redox reactions to change the $\mathrm{pH}$ can also be very low due to the unfavorable surface to volume ratio. An alternative approach can be using redox active molecules like hydroquinone and benzoquinone in the electrolyte that exchange protons [9]. The molecules in contact with the electrode can be oxidized or reduced to change the $\mathrm{pH}$. Using this approach the $\mathrm{pH}$ change close to the electrodes was used to control different chemical reactions but the protons diffuse rapidly, so that the $\mathrm{pH}$ control was not uniform over a large volume. Also, though the working potentials were lower because it avoided the potential drop across several monomer bonds, the problems of electrode degradation and low $\mathrm{pH}$ changes can also be a problem in this case. To increase the surface area and the impact of the redox reactions, Willner and his group reported the electrochemical change of $\mathrm{pH}$ in a confined volume using a three dimensional layer of $\mathrm{Au}$ nanoparticle composites. The composites were electrochemically deposited by crosslinking the thiol functionalized Au nanoparticles with an electrode surface functionalized with SAMs of Aminothiolphenol [10]. The system showed reversible reactions for several cycles with a $\mathrm{pH}$ range of \pm 1 $\mathrm{pH}$ units. A different approach was adopted by Wang et al. to achieve large $\mathrm{pH}$ ranges. Instead of changing the electrodes they constructed a device separated by an interfacial Pd thin film that allowed reduction in one side and oxidation in the other, obtaining opposite changes of $\mathrm{pH}$ in each of the cells [11]. This device achieved a large useful $\mathrm{pH}$ range between 5 and 11 .

The advantage of SAMs to change the acidity is that they can be easily used to obtain highly ordered layers with low redox voltages that can exchange protons in aqueous or organic environments. Aminothiolphenol (ATP) is an organic molecule able to form SAMs in noble metals thanks to the affinity of its mercapto group and the

*Corresponding author: Cesar Pascual-García, Material Research and Technology Department (MRT), Luxembourg Institute of Science and Technology (LIST), 41 Rue du Brill, L-4422 Belvaux, Luxembourg, Europe, Tel: +352 275888 583; E-mail: cesar.pascual@list.lu

Received May 21, 2018; Accepted May 29, 2018; Published June 09, 2018

Citation: Balakrishnan D, Gerard M, Frari DD, Girod S, Olthuis W, et al. (2018) Redox Active Polymer as a pH Actuator on a Re-Sealable Microfluidic Platform. J Material Sci Eng 7: 456. doi: 10.4172/2169-0022.1000456

Copyright: ( 2018 Balakrishnan D, et al. This is an open-access article distributed under the terms of the Creative Commons Attribution License, which permits unrestricted use, distribution, and reproduction in any medium, provided the original author and source are credited. 
interactions among the benzene rings [12,13]. The dissociation constant $\left(\mathrm{pK}_{\mathrm{a}}\right)$ of the amino-group and its interaction with protons has been used to fabricate plasmonic $\mathrm{pH}$ sensors associating the molecule to metal nanoparticles to enhance the vibronic signatures that reveal the protonation state of the molecule [14,15]. ATP can also provide reversible redox active reactions in its polymerized state at low voltages to exchange protons. Though in normal SAMs electrodes the surface volume is very unfavorable within the correct device it can be used to control the $\mathrm{pH}$.

In this paper we review our latest works studying $\mathrm{pH}$ modifications using ATP. We studied different methods of polymerization of ATP including the well-studied electrochemical polymerization and less common ones like photo-polymerization and plasma polymerization attending to the charge transfer, which is the main factor from the SAM's that can influence the $\mathrm{pH}$ change. Then we combined the most efficient method with a device that addresses the challenge miniaturizing the $\mathrm{pH}$ control through the design of a microfluidic platform. The difference with previous works is that in our case we took care that the device could be compatible with miniaturization and the use of small volumes. In this paper we include details of the model and calculations to put an emphasis on the rules we used to obtain a design of a platform that allowed the use of SAMS to change the $\mathrm{pH}$ in more than 6 units in reduced volumes avoiding the degradation of the electrodes. We provide additional details of the fabrication process that we followed to produce chips that are integrated on the platform. Finally, we provide the conclusions and perspectives of such device combined with the improvements of new polymerization methods.

\section{Materials and Methods}

\section{Study of the redox coatings}

Sample preparation to study redox reactions of ATP: Silicon with $50 \mathrm{~nm}$ thermal grown oxide layer was cleaved to obtain $2 \times 1 \mathrm{~cm}^{2}$ samples that were evaporated with Au $30 \mathrm{~nm}$ with an adhesion layer of Ti $5 \mathrm{~nm}$ using an e-beam evaporator. We achieved low roughness $(<2 \mathrm{~nm})$ using an evaporation rate of $0.9 \AA / \mathrm{s}$ at a pressure of $10^{-9} \mathrm{mbar}$. Substrates were then cleaned under a UV ozone lamp for 30 minutes. $0.5 \mathrm{mM}$ concentration of 4 Aminothiolphenol solution in $30 \mathrm{ml}$ of absolute was prepared in a beaker. After the completion of UV ozone cleaning, the samples were placed on the thiol solution beaker and covered with aluminum foil. The beaker was placed under a fumehood for overnight functionalization.

Methods of polymerization: Three different methods of polymerization of ATP were performed, Electropolymerization, Photopolymerization and Plasmapolymerization. Electropolymerization was carried out by cyclic voltammetry using a Princeton applied research Versastat MC potentiostat. The experimental set-up used was a three electrode electrochemical cell with the ATP functionalized Au as working electrode, platinum wire as counter electrode and a calomel reference electrode. Everything was mounted inside a Faraday cage with the beaker with the electrolyte mounted on top of a stage to adjust the height of the exposed working electrode that was fixed to a stand in the Faraday cage. The electrolyte used had $1 \mathrm{M}$ concentration of phosphate buffer solution purged with $\mathrm{N}_{2}$ for five minutes before performing the experiment. The $\mathrm{CV}$ cycles were programmed in the potentiostat with a voltage range between -0.2 to $0.7 \mathrm{~V}$, at $100 \mathrm{mV} / \mathrm{s}$ for 5 cycles.

For photopolymerization or ultra violet polymerization (UV), the substrate functionalized with ATP was placed in a beaker containing 1 $\mathrm{M}$ concentration of phosphate buffer solution. The beaker was placed under a UltraViolet lamp of power $0.9 \mathrm{~mW} / \mathrm{cm}^{2}$ and wavelength of $365 \mathrm{~nm}$ for 30 minutes. Finally, for plasma polymerization, the ATP functionalized substrate was placed on a plane-to-plane direct dielectric barrier discharge plasma generator at atmospheric pressure. Argon plasma with a voltage of $0.4-0.6 \mathrm{kV}$, frequency $10 \mathrm{kHz}$ and a gas flow of $20 \mathrm{~L} / \mathrm{min}$ for two runs is exposed on the substrate.

Electrochemical performance of the polymerized layers: The charge transfer for each polymerization method was calculated from $\mathrm{CV}$ measurements in the same setup and experimental conditions described for the electro polymerization of ATP. The experiments were recorded for the voltage range of -0.2 to $0.7 \mathrm{~V}$ at $100 \mathrm{mV} / \mathrm{s}$ scan rate. The reversibility of the redox reactions were tested by repeating the cycles for up to 50 times.

\section{Implementation in a microfluidic platform}

Fabrication of the electrochemical cell chip for miniaturized control of pH: The electrochemical cell chip was fabricated on a $3 \times$ $3 \mathrm{~cm}^{2}$ Si Substrate with $50 \mathrm{~nm}$ oxide layer using optical lithography (Figure 1). The cell chip consisted of a platinized working electrode (WE) which was connected to a platinized counter electrode (CE) and $\mathrm{Au}$ pseudo reference electrode (RE) each by diffusion barriers (Figure $1 \mathrm{a}$ and $1 \mathrm{~b}$ ). The dimensions of the cell are area of $\mathrm{WE} \mathrm{A}_{\mathrm{we}} \sim 0.05 \mathrm{dm}^{2}$, total area of the cell $\mathrm{A}_{\text {cell }} \sim 0.1 \mathrm{dm}^{2}$, length $\mathrm{L}_{\mathrm{ch}}$ and width $\mathrm{W}_{\mathrm{ch}}$ of the diffusion barrier $\sim 2.6 \mathrm{~cm}$ and $\sim 100 \mu \mathrm{m}$ respectively, the height of the cell $\mathrm{h}=3.5 \mu \mathrm{m}$ and the volume of the cell $\mathrm{V}_{\text {cell }} \sim 120 \mathrm{~nL}$. The complete fabrication process (Figure 1c-1e) we used goes as follows; optical lithography was performed using a maskless laser writer MLA 150 from Heidelberg instruments. Two designs were made using Klayout CAD design program [16]. The first design (M1) included the design of the electrodes with the contact pads. For the M1 design, the substrate was spincoated with LOR 3A (300nm) and S1813 (1.3um) photoresist. Using the laser writer the substrates were exposed with the design M1 with an exposure dose of $120 \mathrm{~mJ} / \mathrm{cm}^{2}$. Then the samples were developed in MF319 solution for 50 seconds and blow dried with $\mathrm{N}_{2}$.
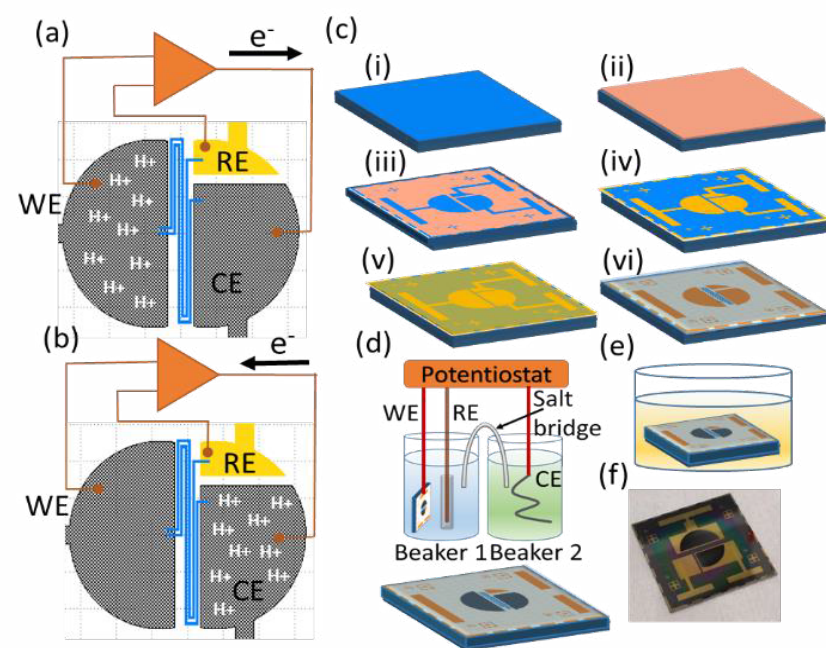

Figure 1: Schematic representation of the electrochemical cell (a) for the oxidation reaction increasing proton concentration in the WE and (b) for the reduction reaction to recover the more basic conditions. (c) Fabrication of the electrochemical cell: a $3 \times 3 \mathrm{~cm}^{2} \mathrm{SiO}_{2} / \mathrm{Si}$ substrate (i) is spin coated with optical resist (ii) the first layer of optical lithography (Layer M1) is defined with laser lithography (iii) and Ti/Au electrodes are e-beam evaporated (iv), second layer of SU8 optical resist is spin coated (v) and optical lithographed (layer M2) (vi) (d), Representation of the Latinization of WE and CE (e) representation of the ATP functionalization. (f) Picture of the fabricated electrochemical chip. 
The photolithographed samples were placed on an e-beam evaporator and $5 \mathrm{~nm}$ Titanium and $50 \mathrm{~nm}$ of Gold were evaporated. The Lift-off process was carried out in a beaker containing $50 \mathrm{ml}$ of acetone. After 15-20 minutes the photo resist S-1813 dissolves leaving the patterned electrodes. The sample was rinsed with distilled water and blow dried with nitrogen. The evaporated sample was again spin coated with SU8 2005 epoxy resist at $5500 \mathrm{rpm}$ and baked at $95^{\circ} \mathrm{C}$ for 1 mins to obtain a uniform layer of $3.5 \mu \mathrm{m}$ thickness. The second design (M2) had the definition of the cell volumes with the diffusion barriers along with the opening for contact pads and electrodes. The substrate was then loaded into the laser writer for the second lithography of design M2 with an exposure dose of $1000 \mathrm{~mJ} / \mathrm{cm}^{2}$. The sample was then baked before developing at $95^{\circ}$ for 3 minutes and then inserted into MICRO-CHEM SU8 developer solution for 1 minute and dried with nitrogen.

Platinisation of electrodes: The experimental set-up (Figure 1d) was as follows: beaker 1 contained $40 \mathrm{mM}$ of chloroplatinic acid and $100 \mu \mathrm{M}$ lead acetate solution in distilled water, beaker 2 contained $1 \mathrm{M}$ of phosphate buffer solution. A salt bridge was made with a filter paper strip that was immersed in potassium chloride solution, after removing the excess of electrolyte it was placed between the two beakers. The salt bridge was immersed in both liquids of the beakers. Using a clamp, the working electrode contact pad of the sample was connected to the potentiostat. The sample was immersed in beaker 1 covering the electrode to be platinized. We used a $\mathrm{Ag} / \mathrm{AgCl}$ reference electrode immersed in beaker 1 . The Pt counter electrode was immersed in beaker 2. A cyclic voltammogram experiment was created using the potentiostat between the voltage range $-0.2 \mathrm{~V}$ to $0.6 \mathrm{~V}$ at a scan rate of $100 \mathrm{mV} / \mathrm{s}$ for 4 cycles. After the completion of the 4 cycles, the sample was removed from the beaker and washed with distilled water, ethanol and blow dried with nitrogen. The counter electrode was also platinized following the same procedure. Then sample was cleaned with ethanol, water and placed under UV lamp for 30minutes. Finally the sample (Figure 1c) was functionalized with ATP by following the same procedure as explained in previous section.

Description of the microfluidic platform: The $\mu$-fluidic platform to hold the chip consisted of two parts with top and the bottom frames (in yellow and grey respectively in Figure 2a-2e. The top frame included a glass window that provided optical access, the $\mu$-fluidic inlet and outlet for the flow of liquids and the electrical contacts. The contacts to the chip were made through springs and they were connected to a switching box allowing the electrodes to be grounded or connected to the potentiostat. The bottom frame included the sample stage with a pneumatic actuator (in blue) using compressed air to lift the chip after the liquid was inserted in the channel, leaving in these way the cells connected only through the electrolyte in the diffusion barriers. The two parts were sealed with an elastic O-ring (Figure 2c) and closed by screws. The chip on the stage (in blue) could be moved up (cell closed) or down (cell open) by opening the valve of the compressed air (V1). In open position, the O-ring pushed down the chip allowing a $60 \mu \mathrm{m}$ gap between the chip and the glass window on the top frame. This position will allow the filling and exchange of liquids in the cell. In closed position the chip was pushed up in contact with the glass window on the top frame and the cells are isolated from $\mu$-fluidic channel and are only connected through diffusion barriers. A syringe with the electrolyte was connected to the $\mu$-fluidic inlet tube (V2) and an empty syringe is connected the $\mu$-fluidic outlet tube (V3). A vacuum pump was connected to the other inlet of T-valve V3, and to ensure the complete filling vacuum was done before inserting the electrolyte.

Optical set-up: In Figure 2f, the optical set-up was built above the platform for optical inspection through the glass window on the top frame. $\mathrm{pH}$ change inside the electrochemical cell was detected using fluorescence dye carboxy semi-naphthorhodafluors (carboxy SNARFs). These molecules are excited using a $565 \mathrm{~nm}$ diode source through a dichoric mirror CROMA-49017 with an excitation window between $540 \mathrm{~nm}$ and $580 \mathrm{~nm}$ and with a low band pass filter at $590 \mathrm{~nm}$ for collection. The excited area was limited by the focusing/collective objective to a field of view smaller than the WE. In order to increase the fluorescence signal all the light collected by the objective was sent to a wide range spectrometer FLAME-S-VIS-NIR. SNARF fluorescence has two peaks at 590 and $650 \mathrm{~nm}$. The peak at $590 \mathrm{~nm}$ is dominant for acidic $\mathrm{pH}$ and $650 \mathrm{~nm}$ peak in dominant for neutral $\mathrm{pH}$, and the ratio between the two peaks can be used to track the $\mathrm{pH}$ in the range from 8 to 5 [17]. The peak at $590 \mathrm{~nm}$ is much weaker than the one at $650 \mathrm{~nm}$, and the signal at high proton concentrations became very weak. For this reason we used the ratio of the two peaks to assess the initial $\mathrm{pH}$
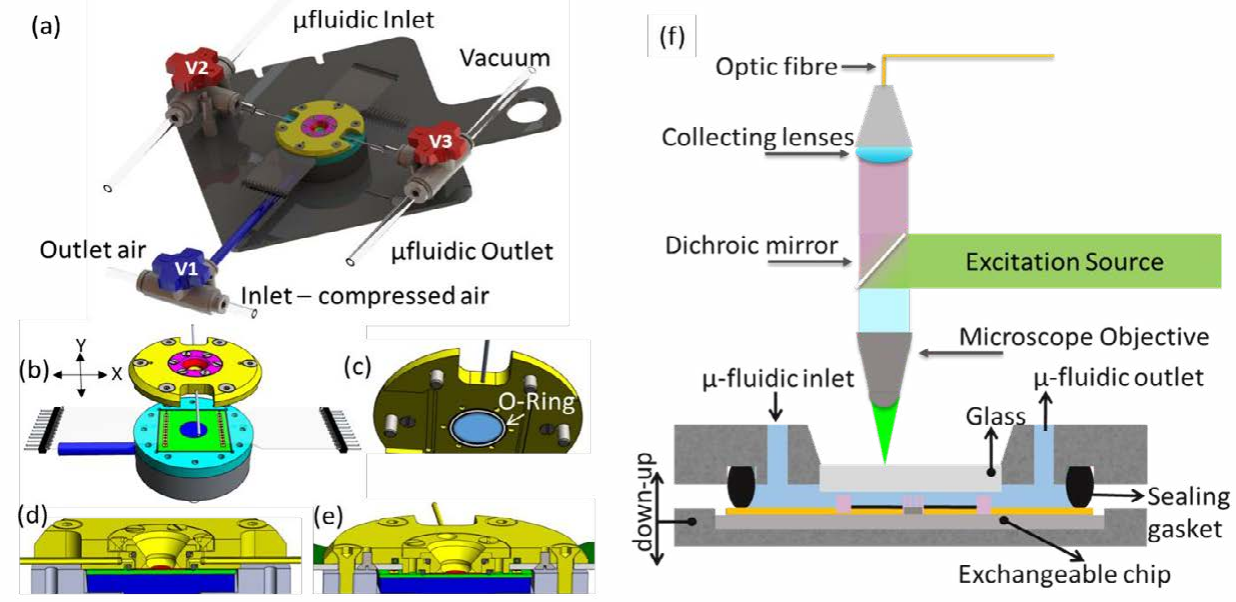

Figure 2: (a) Pictorial representation of the set-up of the microfluidic platform with valves for fluidic transport and pneumatic actuation, (b) opened platform showing the top frame in yellow, bottom frame in grey holding the chip in green. (c) The underlying view of the top frame containing the glass and the O-ring. (d) Cross-section view of the chip mounted on the platform along $X$ axis showing the inlet and outlet for microfluidic transport. (e) The same along Y-axis showing the springs of the electrical contacts on the top to connect the chip. (f) Schematic representation of the microfluidic platform with the optical setup. 
and we followed the total $\mathrm{pH}$ using the $565 \mathrm{~nm}$ excitation. More details about the calibration of SNARF are reported in the supplementary information. The spectra were integrated for 10 seconds.

Electropolymerization and $\mathrm{pH}$ control on the platform: The electropolymersiation was performed with the cell in its opened position. For these experiments and all the others in the platform we used a Solartron Modulab XM Pstat $1 \mathrm{mS} / \mathrm{s}$ potentiostat using three electrode configuration. A cyclic voltammogram was programmed in the poteniostat between of $-0.9 \mathrm{~V}$ to $0.9 \mathrm{~V}$ using a scan rate of $50 \mathrm{mV} / \mathrm{s}$ for 4 cycles.

Before the $\mathrm{pH}$ control experiments, the cell was flushed exchanging the electrolyte between the two syringes in the circuit. The cell was then closed using the valve $\mathrm{V} 1$, leaving the WE communicate to the RE and $\mathrm{CE}$ only through diffusion barriers. A cyclic voltammetry experiment is pre-programmed in the potentiostat for a voltage range of $0.75 \mathrm{~V}$ to $-0.75 \mathrm{~V}$ at a scan rate of $50 \mathrm{mV} / \mathrm{s}$. The fluorescence acquisition was started with before the voltage scan, focusing on the signal from the WE.

\section{Results}

\section{Polymerization and reversibility of redox reactions of ATP}

Figure 3a-3i shows the structural representation of the selfassembled ATP adsorbed on Au, which can polymerized in different ways (Figure 3ii-3iv) depending on the polymerization method. We discussed the different possibilities of polymerization in our previous article [18]. All the three methods provided reversible proton exchange reactions were 2 protons and 2 electrons are exchanged from the molecule to the electrolyte. Figure $2 \mathrm{~b}$ shows representative cyclic voltammograms $(\mathrm{CV})$ of the polymerized samples electropolymerized in black, UV polymerized in red and plasma polymerized in blue. Redox active behavior was observed in all the cases with an oxidation and reduction potential around $\sim 0.2 \mathrm{~V}$ and $\sim 0 \mathrm{~V}$ respectively. The total charge exchange $(Q)$ of these peaks could be calculated from the area withstanding below the redox peaks:

$$
\mathrm{Q}=\frac{1}{v} \int_{\mathrm{V} 1}^{\mathrm{V} 2} \mathrm{I} \cdot \mathrm{dV}-\left(\mathrm{V}_{2}-\mathrm{V}_{1}\right)\left\{\mathrm{I}_{1}+\frac{1}{2}\left(\mathrm{I}_{2}-\mathrm{I}_{1}\right)\right\}
$$

Figure 3a shows a representative example of the limits of the peak chosen to make this calculation.

To study the reversibility of redox reactions of the different polymerization types we repeated $50 \mathrm{CV}$ cycles. In Figure $3 \mathrm{c}$ the charge transfer for the oxidation and reduction peaks are plotted against the number of cycles. We observed that while electropolymerization initially showed a higher charge transfer respect to the other methods and afterwards it degraded to approximately the same value of the other polymerization methods. In the other hand, the charge transfer of the UV and plasma polymerization was nearly constant and shows less degradation with successive cycles. The advantage of electropolymerization is a higher charge transfer at the beginning, however, UV or plasma polymerizations are more suitable for delivering large batches of samples. If the application intends many cycles, they do not have much difference of charge transfer after 50 cycles. More details of the characterization and performance of the polymerized layers can be found in our previous work [18].

\section{Modelling the $\mathrm{pH}$ actuation in the cell}

To model the $\mathrm{pH}$ changes in the cell shown in Figure 1a due to proton exchange reactions like the ones described above, we assume that the charge transfer from the electropolymerized layers corresponds only to proton exchange reactions and that the initial proton concentration in the WE electrode is negligible, which can occur in water if the total change of $\mathrm{pH}$ is several units. We also assume that the protons are confined (and thus they cannot be reduced at the $\mathrm{CE}$ ), which occurs if the diffusion time across the barriers is much longer than the CV cycles.

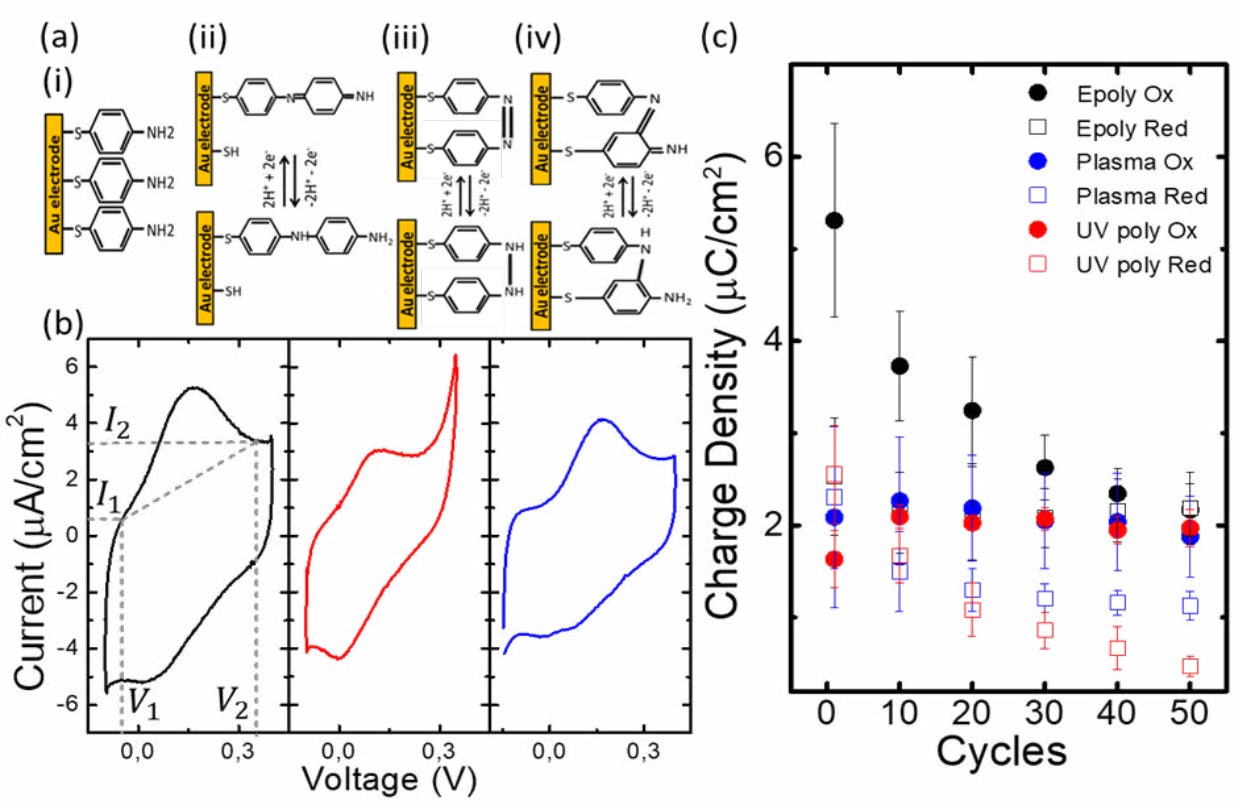

Figure 3: (a) Structural representation of (i) Aminothiolphenol, (ii) electropolymerized, (iii-iv) optical and plasma polymerized SAMs on Au electrodes. (b) Cyclic voltammograms of the polymerized layers where black, red and blue correspond to electrochemical, optical and plasma methods respectively. (c) Charge transfer for oxidation and reduction reactions of ATP for different polymerization methods is plotted against the number of cycles. (Figure adapted from our previous publication in 18). 
The cell in Figure 1a is a 3 electrode electrochemical cell with WE, $\mathrm{CE}$ and RE separated by diffusion barriers. To provide an example of how the cell can control the $\mathrm{pH}$ we will assume that the electrode materials are the ATP coated flat electrodes with same surface coverage as described above. With an applied voltage, the ATP molecules exchange protons with the solution thereby changing the $\mathrm{pH}$ from higher (neutral) (Figure 1b) to lower (acidic) (Figure 1a) and vice versa. The diffusion barrier separates the electrodes into different cells connected by a narrow long channel. In order to avoid diffusion of protons, the length of the barrier was designed extensively longer than the width and height of the barrier and this increase the diffusion time of protons. A barrier length of $2.6 \mathrm{~cm}$ is chosen and for this length, the diffusion time $(t)$ needed for each proton to reach the $\mathrm{CE}$ was calculated using the proton diffusion coefficient $\mathrm{D}=9 \mathrm{E} 10-5 \mathrm{~cm}^{2} / \mathrm{s}$ [19].

$$
\mathrm{t}=\frac{\left(1_{\mathrm{ch}}\right)^{2}}{\mathrm{D}}=130 \text { minutes }
$$

We assume at this point that the total charge involved in the redox process is the one derived from the peak where the limits are indicated in the cyclic voltammogram data of the electropolymerization process in Figure $3 \mathrm{~b}$ and the surface coverage $(S)$ of the ATP molecules is calculated.

$$
\mathrm{S}=\frac{\mathrm{Q}}{\mathrm{A} . \mathrm{F}}=\frac{4.671 \times 10^{-6} \mathrm{Ccm}^{-2}}{96485.33 \mathrm{Cmol}^{-1}}=4.84 \times 10^{-11} \mathrm{~mol} / \mathrm{cm}^{2}
$$

where the area chosen was the footprint of the WE of the chips in in Figure 1 and $F$ is the Faraday constant.

Another factor that contributes to the loss of protons is migration across the barrier. High concentration of electrolyte (1M KCL) is used to avoid migration and to decrease the over potential across the barrier.

After a complete $\mathrm{CV}$ oxidation, the maximum number of protons $(N)$ that can be in solution are the ones corresponding to the surface area of the electrodes. The minimum possible $\mathrm{pH}$ will correspond to the distributions of these protons in the volume of the WE, which can be calculated as follows:

$$
\mathrm{pH}=-\log \left[\frac{\mathrm{N}}{\mathrm{V}_{\text {we }}}\right]=-\log \left[\frac{\mathrm{S} \cdot \mathrm{A}_{\text {we }}}{\mathrm{h} \cdot \mathrm{A}_{\text {we }}}\right]=-\log \left[\frac{\mathrm{S}}{\mathrm{h}}\right]=-\log \left[\frac{4.84 \times 10^{-11} \mathrm{molcm}^{-2}}{3.5 \mu \mathrm{m}}\right]=3.9
$$

where $A_{w e}$ and $V_{w e}$ corresponds to Area and volume of the working electrode respectively and $h$ is the height of the cell. Again for an estimation of the possible $\mathrm{pH}$ with our fabrication parameters we have used the dimensions from the chips in Figure 1 and the data from the electropolymerization in Figure $3 b$.

In order to increase the impact of the electrodes in the $\mathrm{pH}$ we increased the surface area by platinizing, leading to an effective surface charge normalized to the footprint, which was much higher.

\section{pH control experiments on the re-sealable platform}

The reversible states in our chip were achieved through an electropolymerization in the platform. Figure 4 shows the cyclic voltammogram of the electropolymerization process on the chip as a function of voltage (a) and time (b), with reversible oxidation and reduction peaks around $0.2 \mathrm{~V}$ vs the cell gold pseudo reference electrode. During the first rise of current (in black) an increase of the slope after 0.6 $\mathrm{V}$ can be observed that corresponds to the polymerization. This process is similar to what we observed in our previous work [18], although some changes were observed in the value of the voltages because of the pseudoreference electrode and the materials used in the counterelectrode. For each cycle the current density increases. We attribute this effect to additional polymerization of ATP to form redox active molecules, which were not able to polymerize in the first oxidation.

The $\mathrm{pH}$ changes which were simultaneously monitored by measuring the fluorescence at the WE through the SNARF fluorescence marker dissolved in the electrolyte. We used SNARF molecule to monitor the $\mathrm{pH}$ behavior (details of the SNARF fluorescence behavior and calibration is included in supporting information). Figure $4 \mathrm{c}$ shows

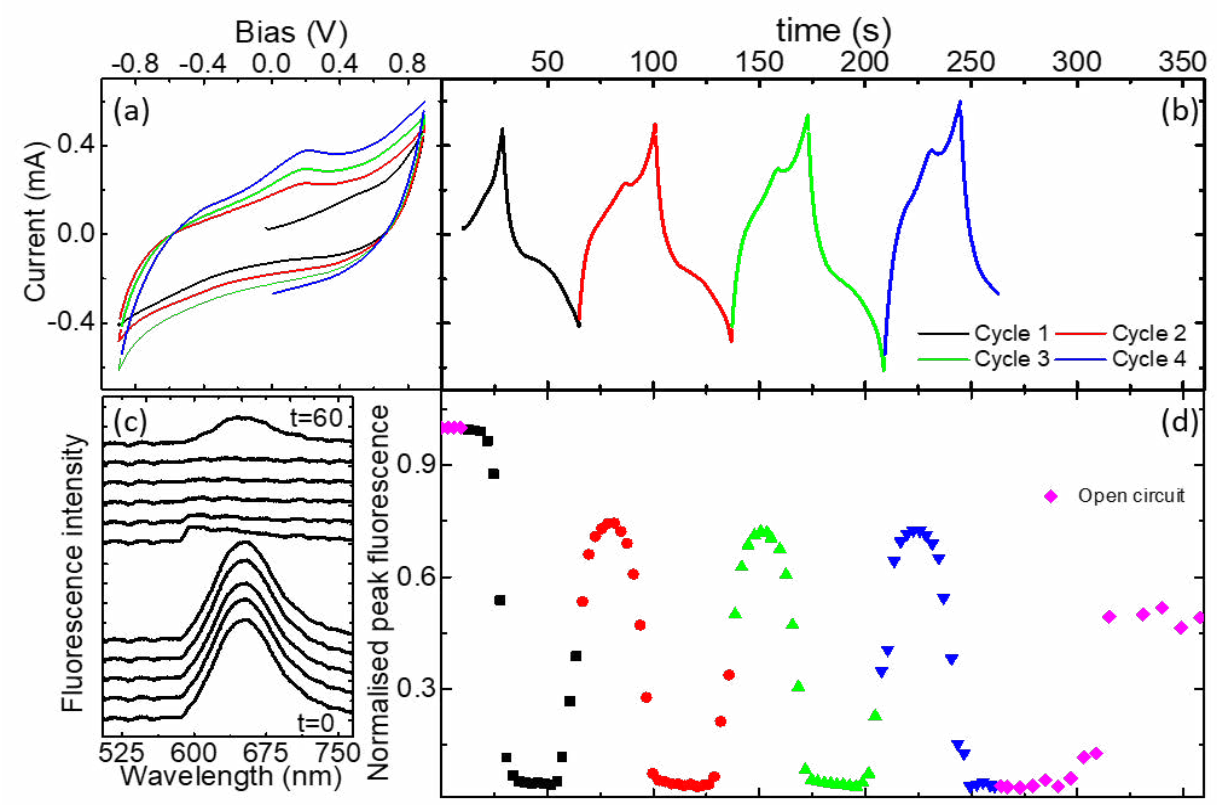

Figure 4: CV of ATP corresponding to the electropolymerization on the chip as a function of voltage (a) and time (b). (c) Sequence of SNARF fluorescence spectra during the first cycle of polymerization. (d) Peak intensity of the fluorescence normalised to its maximum value at neutral $\mathrm{pH}$ as a function of time during the polymerization cycles. 
fluorescence during the first rise in black. SNARF has two peaks at 590 and $655 \mathrm{~nm}$ which are dominant at acidic and basic $\mathrm{pH}$ respectively, however at acidic pH's the overall intensity is much weaker. For this reason we could use the relative intensity of the peaks to verify the initial neutral $\mathrm{pH}$, and then, followed the $\mathrm{pH}$ with the intensity of the $655 \mathrm{~nm}$ peak. In Figure 4c the first four spectra from the bottom correspond to the initial state with neutral $\mathrm{pH}$. In the next spectrum, the 590 peak is more dominant showing that the $\mathrm{pH}$ had decreased. This corresponds to the polymerization and the subsequent oxidation. When the acidity increases the spectra are quenched, indicating that the fluorescence marker is beyond its acidity range $(\sim \mathrm{pH} 5)$. The spectra is recovered after $60 \mathrm{~s}$, in the last spectrum shown after the reduction, which recovers protons into the coating.

In Figure $4 \mathrm{~d}$ the intensity of the $655 \mathrm{~nm}$ is shown versus time for all the cycles. This switch of $\mathrm{pH}$ from higher to lower is repeated with successive cycles. The changes in acidity proved that the redox reactions involved the exchange of protons. These $\mathrm{pH}$ changes were observed because the cell was still only $60 \mu \mathrm{m}$, but the $\mathrm{pH}$ increased with time, as it can be observed by the raise of the fluorescence occurring after the last cycle in blue.

After the polymerization, the cell was closed to observe the $\mathrm{pH}$ changes with confined protons. Figure 5 shows the $\mathrm{CV}$ of 8 cycles representing the proton exchange reactions of ATP in a closed cell. (a) The applied voltage and (b) the measured current with respect to time are plotted. The corresponding SNARF fluorescence spectra is extracted and plotted against time in (c). For each cycle the fluorescence intensity

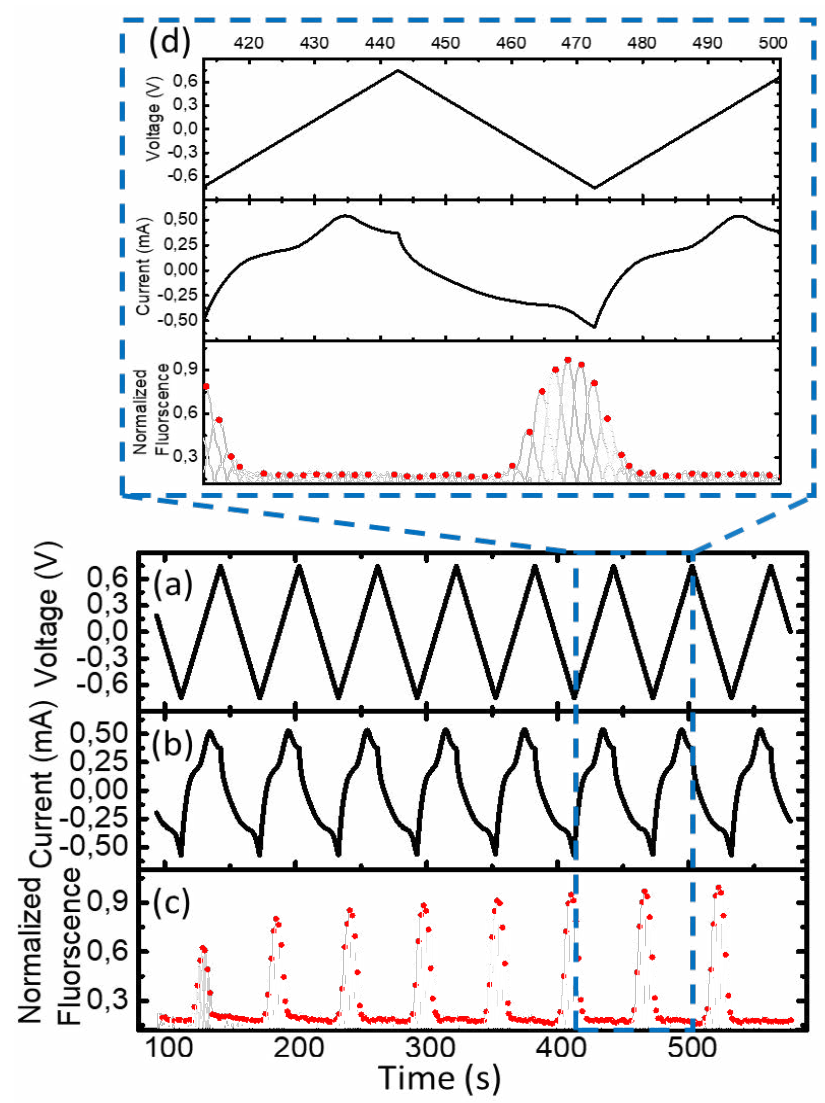

Figure 5: (a) Voltage, (b) current and (c) the intensity of SNARF fluorescence represented as a function of time. (d) The inset above shows the zoomed view of the plot for 1.5 cycle. shifts from maximum to minimum and this process is repeated for successive cycles showing reversible $\mathrm{pH}$ changes. Using the $\mathrm{pH}$ calibration curve of the SNARF fluorescence marker [SI] the maximum and minimum intensity corresponds to $\mathrm{pH} 7.2$ and 5 respectively. The minimum $\mathrm{pH}$ achieved is beyond the detection limit of the SNARF fluorescence marker. Using the CV plot the lowest $\mathrm{pH}$ (using eqn. (1)) was calculated as 0.9. A more detailed information about the SNARF fluorescence marker and CV experiments were reported in our previous publication [20].

In summary, we have presented a molecule, ATP that traditionally was used for sensors thanks to its ability to exchange protons, but that in a smart microfluidic device, we have been able to use it to change the $\mathrm{pH}$ in nanoliter volumes. We have reviewed the routes to obtain reversible redox states by polymerizations, and the efficiency of the redox active reactions achieved. A miniaturized electrochemical cell of few $100 \mathrm{~nL}$ was modelled to control the $\mathrm{pH}$. The detailed steps involved in the fabrication process and the design of the platform are explained. We have presented how to control critical parameters involved in the design of the cell like diffusion time of protons through the barrier, dimensions of the cell and the estimation of minimum $\mathrm{pH}$ that could be achieved with flat electrodes. We have demonstrated a setup involving our platform to monitor the $\mathrm{pH}$ changes in these volumes, in the electropolymerization and in the $\mathrm{pH}$ control experiments.

The cell that we have presented is compatible with miniaturization. Eqn. (4) shows that the minimum $\mathrm{pH}$ does not depend on the area of the electrodes, thus different designs could be implemented depending only on the effective surface coverage of ATP and the height of the cell in its closed position. This opens the way to new designs that could include further nanostructuration of the electrodes, other than platinisation to control the effective surface area in a rational way. The single channel architecture of our cell is also compatible with the inclusion of several cells, which would allow multiplexing of $\mathrm{pH}$ control in a single device. For these novel implementations, the presented methods of polymerization provide flexibility for mass production outside the chamber, and post modification of the electrodes for example ion permeable layers.

In conclusion, we believe that our platform can open the way to the control of chemical reactions through the systematic control of proton concentration, allowing miniaturization and multiplexing.

\section{Acknowledgement}

This investigation was financed by the FNR ATTRACT project 5718158 $\mathrm{NANOpH}$.

\section{References}

1. Eckermann AL, Feld DJ, Shaw JA, Meade TJ (2010) Electrochemistry of redoxactive self-assembled monolayers. Coord Chem Rev 254: 1769-1802.

2. Penner RM (2010) Electrochemistry: Protons on tap. Nat Chem 2: 251-252.

3. Gao X, Yu P, LeProust E, Sonigo L, Pellois JP, et al. (1998) Oligonucleotide synthesis using solution photogenerated acids. J Am Chem Soc 120: 1269812699.

4. Cleland WW (1982) The use of $\mathrm{pH}$ studies to determine chemical mechanisms of enzyme catalyzed reactions. Methods in Enzymology 87: 390-405.

5. Chunl BS, Jhona MS, Leeb HB, Yukb SH (1998) pH/temperature dependent phase transition of an interpenetrating polymer network: anomalous swelling behavior above lower critical solution temperature. Eur Polym J 34: 1675-1681.

6. Morimoto K, Toya M, Fukuda J, Suzuki H (2008) Automatic Electrochemical Micro-pH-Stat for Biomicrosystems. Anal Chem 80: 905-914.

7. Shinohara H, Kojima J, Aizawa M (1989) Electrically controlled ion transfer and 
Citation: Balakrishnan D, Gerard M, Frari DD, Girod S, Olthuis W, et al. (2018) Redox Active Polymer as a pH Actuator on a Re-Sealable Microfluidic Platform. J Material Sci Eng 7: 456. doi: 10.4172/2169-0022.1000456

Page 7 of 7

$\mathrm{pH}$ change near a conducting polymer-coated electrode. J electroanal Chem Interfacial Electrochem 266: 297-308

8. Lefebvre MC, Qi Z, Pickup PG (1999) Electronically Conducting Proton Exchange Polymers as Catalyst Supports for Proton Exchange Membrane Fuel Cells. Electrocatalysis of Oxygen Reduction, Hydrogen Oxidation, and Methanol Oxidation. J Electrochem Soc 146: 2054-2058.

9. Maurer K, Cooper J, Caraballo M, Crye J, Suciu D, et al. (2006) Electrochemically generated acid and its containment to 100 micron reaction areas for the production of DNA microarrays. PLoS One 1: e34.

10. Frasconi M, Tel-Vered R, Elbaz J, Willner I (2010) Electrochemically Stimulated pH Changes: A Route To Control Chemical Reactivity. J Am Chem Soc 132: 2029-2036.

11. Wang YC, Lin CB, Su JJ, Ru YM, Wu Q, et al. (2011) Electrochemically driven large amplitude $\mathrm{pH}$ cycling for Acid-Base driven DNA denaturation and renaturation. Anal Chem 83: 4930-4935.

12. Cho SH, Kim D, Park SM (2008) Electrochemistry of conductive polymers. Effects of self-assembled monolayers of aminothiolphenols on polyaniline films. Electrochimica Acta 53: 3820-3827.

13. Ahao LB, Zhang M, Ren B, Tian ZQ, Wu DY (2014) Theoretical study on thermodynamic and spectroscopic properties of electro-oxidation of
p-Aminothiolphenol on Gold electrode surfaces. J Phys Chem C 118: 27113 27122.

14. Zong S, Wang Z, Yang J, Cui Y (2011) Intracellular pH Sensing Using p-Aminothiophenol Functionalized Gold Nanorods with Low Cytotoxicity. Ana Chem 83: 4178-4183.

15. Bishnoi SW, Rozell CJ, Levin CS, Gheith MK, Johnson BR, et al. (2006) AllOptical Nanoscale pH Meter. Nano Lett 6 (8): 1687-1692.

16. www.klayout.de

17. Johnson ID (2010) Molecular probes handbook. Life Technologies Corporation 11: Chapter 20.

18. Balakrishnan D, Lamblin G, Thomann JS, Guillot J, Duday D, et al. (2017) Influence of polymerization on the reversibility of low-energy proton exchange reactions by Para-Aminothiolphenol. Sci Rep 7: 15401.

19. Cussler EL (1997) Diffusion: Mass transfer in fluid systems. Cambridge University press.

20. Balakrishnan D, Lamblin G, Thomann JS, van den Berg A, Olthuis W, et al (2018) Electrochemical control of pH in nano-litre volumes. Nano Lett 18: 2807 2815. 